\title{
Making efficient public good decisions using an augmented Ausubel auction
}

\author{
Matt Van Essen
}

Received: 9 March 2013 / Accepted: 13 March 2013 / Published online: 25 March 2013

(C) SAET 2013

\begin{abstract}
We provide the generalization of Ausubel's 2004 ascending bid auction to public good environments. Like its private good counterpart, the public good Ausubel auction encourages truthful revelation of preferences, is privacy preserving, and yields an equilibrium allocation that is outcome equivalent to the public good Vickrey auction. Other properties are not ideal in a public good setting. We discuss two such issues and propose an alternative dynamic auction which solves these problems.
\end{abstract}

Keywords Public goods $\cdot$ Clarke tax $\cdot$ Ausubel auction

JEL Classification $\quad \mathrm{C} 72 \cdot \mathrm{D} 44 \cdot \mathrm{H} 41$

\section{Introduction}

After almost 50 years of research on incentive design, it remains unclear whether a practical institution can be designed to overcome free-riding incentives in a public good environment. While this problem is easier if agents reveal their preferences to a decision maker, agents' interests are not typically aligned with those of the decision maker. As a result, agents may misrepresent their preferences. In this paper, we look to recent contributions in dynamic private good auctions and experimental economics to

This paper has benefited from the many helpful discussions I had with John Wooders. I am also grateful to Mark Walker, Rabah Amir, Mark Stegman, Martin Dufwenberg, Ron Harstad, two anonymous referees, and an associate editor for their helpful comments.

M. V. Essen ( $\varangle)$

Department of Economics, Finance, and Legal Studies, University of Alabama, Tuscaloosa, USA

e-mail: mjvanessen@cba.ua.edu 
give us insight into a new, and hopefully improved, way of overcoming the "preference revelation" problem in a public good setting.

Early work in this area came from Vickrey (1961) in a private good setting. He proposed an auction which efficiently allocates multiple units of a homogeneous good by encouraging revelation of preferences as a dominant strategy. His mechanism was later generalized by Clarke (1971) and Groves (1973) to accommodate public goods. Loeb (1977) shows the Vickrey auction can also be re-defined to make efficient public good decisions. These mechanisms are known as VCG mechanisms and have generated a large literature. ${ }^{1}$ Despite the theoretical interest in VCG mechanisms, Rothkopf (2007), and others, have argued they are not practical due to lack of privacy preservation. Intuitively, an auction is privacy preserving for a bidder if, when the auction ends, the seller cannot construct a complete demand schedule for that bidder. A second price sealed bid auction, for example, is not privacy preserving since at the end of the auction a seller knows all the valuations. This feature encourages practices like 'shill' bidding and may dissuade bidders from participating. Privacy preservation is also a concern in public good settings, where consumers prefer the government not know their true valuations.

A potential solution to this critique is to use a dynamic auction to make allocation decisions. A Japanese auction, for example, is outcome equivalent to the second price sealed bid auction, but preserves the privacy of the winning bidder. In this auction, an auctioneer slowly raises the price and bidders signal each round whether they want to continue and drop out if the price becomes too high. The last bidder still in the auction wins and pays the price the second to last bidder dropped out at. However, unlike the second price auction, the winner never reveals his maximum willingness to pay. Thus, in a dynamic setting the auction can stop before bidders can reveal their whole valuation schedule. Ausubel (2004) introduced a dynamic auction that retains the nice revelation properties of the private good multi-unit Vickrey auction, is outcome equivalent to the Vickrey auction, and preserves the privacy of some of the bidders. ${ }^{2}$ In addition, this auction has been more "behaviorally" successful than its sealed bid counterpart when tested in the laboratory. Kagel and Levin (2001) find that bidders in the private good Ausubel auction do, in general, bid truthfully when compared with bidders in uniform price sealed bid auctions. In a follow up paper, Kagel and Levin (2001) compare the private good, multi-unit Vickrey auction against the private good Ausubel auction with different information feedback treatments finding the Ausubel auction outperforms the Vickrey auction. ${ }^{3}$ The authors credit these results to the relative transparency of Ausubel's auction.

We define and study the public good Ausubel auction. The intuition for the transition is simple. In the private good Ausubel setting, all bidders take the auctioneer's price

\footnotetext{
1 There are important differences between VCG mechanisms. To avoid confusion we refer to authors (i.e., Vickrey or Clarke) when refering to a specific VCG mechanism.

${ }^{2}$ Bergemann and Välimäki (2010) is a dynamic treatment of the VCG mechanism in an infinite horizon environment.

${ }^{3}$ See also Kagel et al. (2007).
} 
as given and respond with (potentially) different quantity bids. ${ }^{4}$ In a public good setting, bidders face a uniform quantity of the public good, by definition, but have (potentially) different marginal valuations for each unit. Thus, it is natural to re-define the private good Ausubel auction by using an ascending "quantity" auction instead of an ascending "price" auction. In this new mechanism, the auctioneer starts by calling out a low quantity (instead of a price) and individuals respond by submitting value bids (instead of quantity bids). If the bids exceed the marginal cost (i.e., supply for that unit), the auction continues and the auctioneer increases the quantity. This process continues until the sum of the bids no longer exceeds the marginal cost-i.e., until there is no longer excess inverse demand for the public good. Truth telling is made incentive compatible by adopting a variation of Ausubel's "clinching rule" to determine the individuals' taxes.

The resulting auction is the ascending quantity Ausubel auction (AQ-AA) and it has a number of nice features. We show the AQ-AA is privacy preserving, that truthful revelation of bidders' valuations is supported as an equilibrium in a variety of strategic environments, and that auction is outcome equivalent to the public good Vickrey auction. However, not all of the properties of the AQ-AA are ideal in a public good setting. We conclude the paper by discussing two shortcomings of the AQ-AA that do not appear in the private good Ausubel auction and introduce a dynamic auction with similar strategic properties that corrects these problems.

\section{The public good economy}

The setting consists of $N \geq 2$ bidders that participate in an auction to determine the production level of a public good $x$. The set of production levels is $X=\{0,1, \ldots, \bar{x}\}$, where the total production cost of $x$ is $c(x)=c x$, where $c \in \mathbb{R}_{+}$. For each unit $m>0$ produced, there is a reservation price $\theta_{i}^{m} \in\{0, \epsilon, 2 \epsilon, \ldots\}$ that indexes each bidder $i$ 's maximum willingness to pay, where $\epsilon>0$ is the minimum increment. We assume reservation prices are weakly decreasing in the public good, where an arbitrary profile of $\bar{x}$ reservation prices for bidder $i$ is $\theta_{i}=\left(\theta_{i}^{1}, \ldots, \theta_{i}^{\bar{x}}\right)$. The set of all such profiles is $\Theta_{i}$, where $\Theta=\times_{i=1}^{N} \Theta_{i}$. Types are independently drawn before the auction begins (i.e., at stage 0 ) according to the distribution $f_{i}$, where each bidder observes only his own type profile. The joint distribution is denoted by $f$. Bidders preferences are represented by a quasi-linear payoff function $u_{i}: X \times \Theta \times \mathbb{R}_{+} \rightarrow \mathbb{R}$ which is equal to his private value, $v_{i}\left(x, \theta_{i}\right)$, for the quantity $x$ of the public good produced less the total tax payment $\tau_{i}$-i.e., $u_{i}\left(x, \tau_{i}, \theta\right)=v_{i}\left(x, \theta_{i}\right)-\tau_{i}$, where

$$
v_{i}\left(x, \theta_{i}\right)= \begin{cases}0 & \text { if } x=0 \\ \sum_{j=1}^{x} \theta_{i}^{j} & \text { otherwise }\end{cases}
$$

An allocation $\left(x, \tau_{1}, \ldots, \tau_{N}\right) \in X \times \mathbb{R}_{+}^{N}$ determines the level of the public good produced and a total payment for each bidder. We are interested in allocations that

\footnotetext{
${ }^{4}$ Since this price uniformly applies to all bidders in a private good setting, it can be thought of as a public good.
} 
make efficient public good decisions - i.e., $\left.\hat{x} \in \arg \max _{x \in X}\left[\sum_{i=1}^{N} v_{i}\left(x, \theta_{i}\right)-c(x)\right]\right)$. Finally, two additional assumptions are used in some of our results:

- Bounded Type Assumption (BTA) No bidder type prefers to unilaterally finance production of the public good-i.e., $0 \leq \theta_{i}^{1} \leq c-\epsilon$ for all $i$.

- Bounded Marginal Cost Assumption (BMCA) The marginal cost satisfies $c \leq$ $(N-1)(c-\epsilon)$.

\section{The public good Vickrey auction}

In the Vickrey auction, bidders submit demand schedules to the government, who chooses the level of the public good that maximizes social surplus and determines a tax payment for each bidder. ${ }^{5}$ Specifically, each bidder $i$ sends a bid $b_{i} \in \Theta_{i}$ to the government. Let $b \in \Theta$ be an arbitrary bid profile and $b_{-i} \in \times_{j \neq i} \Theta_{j}$ be an arbitrary bid profile by bidders other than $i$. The government takes the bids as proxies for bidders' valuation schedules and constructs a reported valuation function $\tilde{v}_{i}$, for each $i$, where

$$
\tilde{v}_{i}\left(x, b_{i}\right)=\left\{\begin{array}{ll}
0 & \text { if } x=0 \\
\sum_{j=1}^{x} b_{i}^{j} & \text { otherwise }
\end{array} .\right.
$$

The public good level chosen is $\left.\hat{x}(b) \in \arg \max _{x \in X}\left[\sum_{i=1}^{N} \tilde{v}_{i}\left(x, b_{i}\right)-c(x)\right]\right)$. Last, for each $x$, define $i$ 's residual supply function $\tilde{s}_{i}: X \times B_{-i} \rightarrow \mathbb{R}$, where $\tilde{s}_{i}\left(x, b_{-i}\right)=\max \left\{0, c-\sum_{j \neq i} b_{j}^{x}\right\}$. For each unit produced, the government charges each bidder $i$ a tax equal to their reported residual supply or

$$
\tau_{i}\left(x^{*}, b_{-i}\right)= \begin{cases}0 & \text { if } x^{*}=0 \\ \sum_{j=1}^{x^{*}} \tilde{s}_{i}\left(j, b_{-i}\right) & \text { otherwise. }\end{cases}
$$

The Vickrey auction induces a game such that truth telling is always a best response and under some restrictions is a weakly dominant strategy for all bidders. ${ }^{6}$

Theorem 1 In a public good Vickrey auction with non-decreasing marginal cost, it is always a best response for each bidder to report his true type profile. Furthermore, if the BTA and BMCA are satisfied, then truth telling is a weakly dominant strategy for each bidder.

As an example, suppose the government uses a Vickrey auction to decide how much $x$ to produce at a marginal cost of $\$ 10$ per unit. Bidders' values for $x$ are as follows: $\theta_{A}=(14,4,2), \theta_{B}=(4,3,2)$, and $\theta_{C}=(3,2,1)$. The efficient quantity to produce is one. If bidders $A, B$, and $C$ submit their true type vectors, the auction specifies one unit be produced. Bidder $A$, whose reported residual supply is $\left(\tilde{s}_{A}\left(1, b_{-A}\right), \tilde{s}_{A}\left(2, b_{-A}\right), \tilde{s}_{A}\left(3, b_{-A}\right)\right)=(3,5,7)$, pays a tax equal to $\tilde{s}_{A}\left(1, b_{-A}\right)=3$.

\footnotetext{
5 As there may be multiple maximizing arguments, we shall assume the government chooses the largest $x$.

6 See Loeb (1977) or Milgrom (2004) p. 50-51.
} 


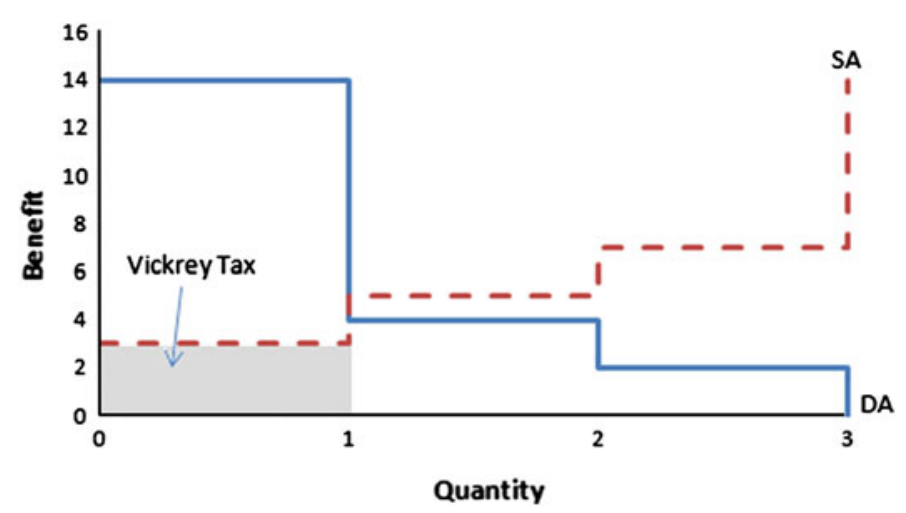

Fig. 1 Revealed bids and tax for a public good Vickrey auction

This is illustrated in Fig. 1. The Vickrey taxes for the other bidders are both zero leaving a total tax revenue of 3 and a budget deficit of $7 .^{7}$

\section{The public good Ausubel auction}

The AQ-AA is a dynamic auction that occurs over rounds. At each round $t=1, \ldots, \bar{x}$, the level of the public good is set to $x=t$ and each bidder $i$ is required to submit a bid $b_{i}^{t} \in B_{i}^{t}$ to the government, where $B_{i}^{t}$ is the set of feasible bids for bidder $i$ at round $t .^{8}$ If $\sum_{i} b_{i}^{t} \geq c$, the auction continues to the next round $(x=t+1)$ and it stops otherwise. Denote the round the auction stops by $L$ so the quantity produced is $x^{*}=L-1$. Bidder $i$ 's residual supply function at round $t$ is a function of the other players' bids (i.e., $b_{-i}^{t} \in B_{-i}^{t}$ ) and is defined by $\tilde{s}_{i}: X \times B_{-i}^{t} \rightarrow \mathbb{R}$, where $\tilde{s}_{i}\left(t, b_{-i}^{t}\right)=\max \left\{0, c-\sum_{j \neq i} b_{j}^{t}\right\}$. At each round $t$ where $b_{i}^{t} \geq \tilde{s}_{i}\left(t, b_{-i}^{t}\right)$, bidder $i$ accrues a tax equal to $\tilde{s}_{i}\left(t, b_{-i}^{t}\right)$. His total payment at the end of the auction is

$$
\tau_{i}\left(L, b_{-i}\right)=\left\{\begin{array}{ll}
0 & \text { if } L=1 \\
\sum_{t=1}^{L-1} \tilde{s}_{i}\left(t, b_{-i}^{t}\right) & \text { otherwise }
\end{array},\right.
$$

where $b_{-i}=\left(b_{-i}^{1}, \ldots, b_{-i}^{L}\right) .^{9}$

Reconsider the previous example using the AQ-AA rules and suppose A, B, and $\mathrm{C}$ bid truthfully. In round $1, \mathrm{~A}, \mathrm{~B}$, and $\mathrm{C}$ submit the bids $\left(b_{A}^{1}, b_{B}^{1}, b_{C}^{1}\right)=(14,4,3)$. Since $14+4+3>c=10$ the auction continues. Bidder $A$ pays $s_{A}(1)=3$ and the other two pay 0 . In round 2 , the quantity is increased to 2 and $A, B$, and $C$ submit bids

\footnotetext{
7 The revenue generated in the public good Vickrey auction always runs a deficit—see Loeb (1977).

8 We define this set more formally in the next section.

9 Interestingly, the AQ-AA is similar to a family of planning procedures initially studied by Malinvaud (1971) and Dreze and Pousin (1971). Often referred to as MDP processes, these mechanisms can be adopted to converge to any pareto optimal outcomes through different divisions of the social surplus. However, they are vulnerable to strategic manipulation.
} 


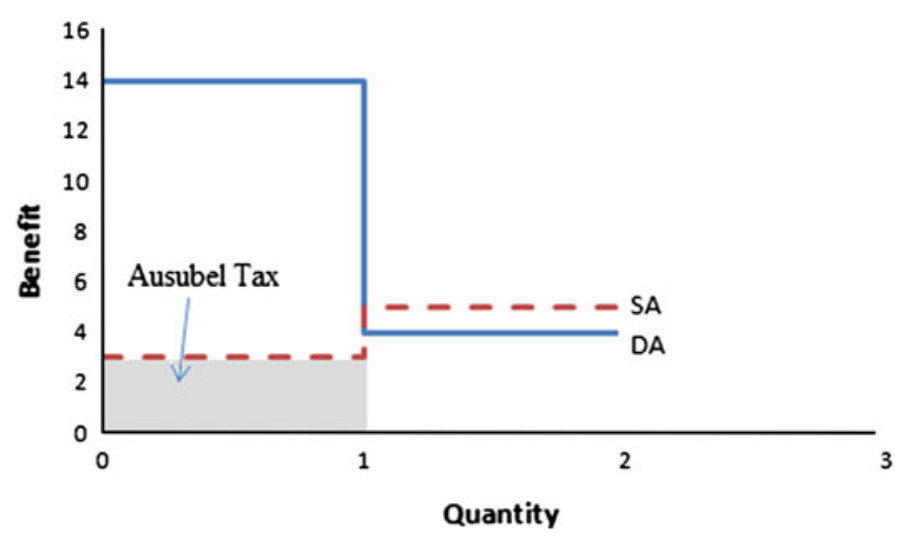

Fig. 2 Revealed bids and tax for an ascending quantity Ausubel auction

$\left(b_{A}^{2}, b_{B}^{2}, b_{C}^{2}\right)=(4,3,2)$. Since $4+3+2<10$, the auction stops. None of the bidders pay a tax in the second round and the final quantity produced is 1 . The total tax revenue is 3 and the outcome is equivalent to the one in the Vickrey auction. The example also shows the AQ-AA preserves the privacy of all three bidders, but it remains to prove truth telling is an equilibrium (Fig. 2).

\subsection{Strategic analysis of the AQ-AA}

The AQ-AA induces a dynamic stage game. In each of the $T$ possible stage, bids are made and the auction progresses or stops according to the rules of the auction. Possible bidding histories at stage $t$ are defined by

$$
H^{t}= \begin{cases}\{\emptyset\} & \text { if } t=1 \\ \left\{\left(b^{1}, \ldots, b^{t-1}\right) \in \times_{m=1}^{t-1} B^{m}\right\} & \text { otherwise }\end{cases}
$$

where $h^{t}$ is an arbitrary element. The set of feasible bids for bidder $i$, at stage $t$ given $h^{t}$, is $B_{i}^{t}\left(h^{t}\right)$. We abuse notation and define $B_{i}^{t}\left(H^{t}\right)=\bigcup_{h^{t} \in H^{t}} B_{i}^{t}\left(h^{t}\right)$. Histories which end the auction are collected in the set $Z$. These histories determine an allocation $\left(x, \tau_{1}, \ldots, \tau_{N}\right)$. Thus, a bidder $i$ 's payoff function $u_{i}$ is defined on these terminal histories and his type. Finally, at each stage $t$, each bidder $i$ has information about the bidding history $h^{t}$. They know their own bid and are given information about the bids of the others according to some "feedback" rule $g_{i}\left(b^{t}\right)$. The set of bid history information for $i$ at stage $t$ is $H_{i}^{t}$, where

$$
H_{i}^{t}= \begin{cases}\{\emptyset\} & \text { if } t=1 \\ \left\{\left(\left(b_{i}^{k}, g_{i}\left(b^{k}\right)\right)\right)_{k=1}^{t-1} \mid h^{t} \in H^{t}\right\} & \text { otherwise }\end{cases}
$$

and $h_{i}^{t}$ is an element of this set. Common rules such as "full bid information," "aggregate bid information," and "no bid information" are defined by the feedback rules 
$g_{i}\left(b^{k}\right)=b_{-i}^{k}, g_{i}\left(b^{k}\right)=\sum_{j \neq i} b_{j}^{k}$, and $g_{i}\left(b^{k}\right)=\left\{1\right.$ if $\sum b_{m} \geq c^{m}$ and 0 otherwise $\}$ respectively. The dynamic game $\Gamma^{e}$ induced by the AQ-AA is therefore defined by

$$
\Gamma^{e}=\left(N, T, f, Z,\left(\Theta_{i},\left(H_{i}^{t}, B_{i}^{t}(\cdot)\right)_{t=1}^{\mathrm{T}}, u_{i}\right)_{i \in N}\right) .
$$

A pure strategy $\beta_{i}$ for bidder $i$ in the game $\Gamma^{e}$ is a collection of functions (one for each stage), where each function maps a bidder's history and type into a feasible action.

If the realization of types in the AQ-AA is common knowledge to all of the bidders, then $\Gamma^{e}$ reduces to a game of complete information. Specifically, for any $\hat{\theta} \in \Theta$, the realized game of complete information $\Gamma^{e}(\hat{\theta})$ is defined by

$$
\Gamma^{e}(\hat{\theta})=\left(N, T, Z,\left(\left(H_{i}^{t}, B_{i}^{t}(\cdot)\right)_{t=1}^{\mathrm{T}}, \hat{u}_{i}(\cdot, \hat{\theta})\right)_{i \in N}\right),
$$

where, for each $i, \hat{u}_{i}(\cdot, \hat{\theta})=u_{i}(\cdot, \hat{\theta})$. A strategy $\delta_{i}$ for a bidder $i$ in $\Gamma^{e}(\hat{\theta})$ is an ex-post pure strategy. For any realization of types $\hat{\theta}$, a strategy profile $\beta$ in $\Gamma^{e}$ can be projected into an ex-post pure strategy profile of the realized game $\Gamma^{e}(\hat{\theta})$ by setting $\delta_{i}^{t}\left(h_{i}^{t}\right)=$ $\beta_{i}^{t}\left(h_{i}^{t}, \hat{\theta}_{i}\right)$ for each $t$. A strategy profile $\beta$ is an ex-post (perfect) Nash equilibrium of $\Gamma^{e}$ if, for each $\theta \in \Theta$, the ex-post pure strategy profile $\delta$ is a (subgame perfect) Nash equilibrium of the game $\Gamma^{e}(\theta)$, where $\delta_{i}^{t}\left(h_{i}^{t}\right)=\beta_{i}^{t}\left(h_{i}^{t}, \theta_{i}\right)$ for each $i, t$, and $h_{i}^{t}{ }^{10}$ Our first result is that truthful bidding behavior is an ex-post perfect Nash equilibrium of the AQ-AA in the full bid information setting - all of the proofs are in the Appendix.

Theorem 2 In the full bid information $A Q-A A$, truth telling by each bidder is an expost perfect Nash equilibrium. Moreover, the truth telling equilibrium allocation of the AQ-AA is outcome equivalent to the truth telling equilibrium of the public good Vickrey auction.

The following corollary follows almost directly.

Corollary 1 In both the aggregate bid information $A Q-A A$ and the no bid information $A Q-A A$, truth telling by each bidder is an ex-post perfect Nash equilibrium.

Ex post perfect Nash equilibrium is not as strong as weak dominance. In fact, truth telling in the AQ-AA with full bid information is not a dominant strategy. To illustrate, consider a two bidder case where bidders $A$ and $B$ have the degenerate type profiles $\theta_{A}=(5,4), \theta_{B}=(4,3)$ respectively, can bid any positive integer, and marginal cost is $c=6$. Consider the strategy profile $\beta=\left(\beta_{A}, \beta_{B}\right)$ defined by:

$$
\begin{aligned}
& \beta_{A}=\left(5, \beta_{A}^{2}\left(h_{A}\right)\right), \quad \text { where } \quad \beta_{A}^{2}\left(h_{A}\right)=4 \text { for all } h_{A} \\
& \beta_{B}=\left(4, \beta_{B}^{2}\left(h_{B}\right)\right), \quad \text { where } \quad \beta_{B}^{2}\left(h_{B}\right)= \begin{cases}0 & \text { if } b_{A}^{1}=5 \\
3 & \text { otherwise. }\end{cases}
\end{aligned}
$$

Given $B$ 's history dependent strategy, $A$ 's truth telling strategy is not a best response. In particular, $A$ 's payoff from truth telling is 3 . He does better switching to strategy

\footnotetext{
10 See, for example, Cremer and McLean (1985) or Holmstrom and Myerson (1983). Ausubel uses a related concept called ex post perfect equilibrium.
} 
$\tilde{\beta}_{A}=\left(4, \beta_{A}^{2}\left(h_{A}\right)\right)$, where $\tilde{\beta}_{A}^{2}\left(h_{A}\right)=4$ for all $h_{A}$. His payoff is now 4 since 2 units of the public good are produced and his residual supply is $s_{A}=(2,3)$. However, if we restrict bids to be decreasing and the amount of information each bidder receives, truth telling is weakly dominant. If we provide "no bid information" and restrict bids to be decreasing in the example above, then each strategies specify a bid for each round contingent on the auction continuing and takes the form $\beta_{i}=\left(b_{i}^{1}, b_{i}^{2}\right)$, where $b_{i}^{1} \geq b_{i}^{2}$. These are just the types of strategies used in the Vickrey auction. It is not surprising therefore that truth telling is weakly dominant. This holds in general.

Theorem 3 If bids are restricted to be weakly decreasing, then truthful revelation of bidding type in each round is always a best response to any bidding strategies of rival bidders in the "no bid information" AQ-AA. Furthermore, if the BTA and BMCA are satisfied, then truth telling is a weakly dominant strategy.

The robustness of the truth telling strategy profile as an equilibrium along with the privacy preservation property make the AQ-AA an appealing procedure. However, despite these features, the auction has two obvious shortcomings. First, it does not generate enough tax revenue to finance production-a major difficulty in application. Second, the AQ-AA protects the marginal valuations for later units in the auction (i.e., the low marginal valuations). This is unfortunate since it is presumably the high marginal valuations (i.e., valuations for earlier units) which the bidders would prefer that the government not know. In the next section, we provide an alternative auction which solves these two problems.

\section{The descending Clarke-Ausubel auction}

We formulate a decreasing quantity auction with strategic properties similar to the AQAA, but which protects the highest marginal valuations for the bidders and covers the cost of production. This mechanism, dubbed the decreasing quantity Clarke-Ausubel auction (DQ-CAA), is motivated by the familiar "Clarke tax." The auction begins at stage 1 where the public good is initially set at $x=\bar{x}$. The cost of the allocation $\bar{x}$ is $C(\bar{x})$. The government imposes an tentative cost sharing rule $\left(s_{1}, \ldots, s_{N}\right)$, where $i$ 's tentative fixed cost share is $s_{i} C(\bar{x})$ for $s_{i} \geq 0$ and $\sum_{i} s_{i}=1$. At each round $t=1, \ldots, \bar{x}+1$, each $i$ submits $b_{i}^{t} \in B_{i}^{t}$ to the government who uses the following continuation rule: If there exist a bidder $i$ such that $\sum_{j \neq i} b_{j}^{t} \leq\left(1-s_{i}\right) c$, then the auction continues to round $t+1$, otherwise the auction stops. Bids are constrained to be weakly increasing. The quantity $x$ produced is determined by the first round $t$ where $\sum_{i} b_{i}^{t} \geq c-$ i.e., $x=\bar{x}-(t-1)$. Finally, at each stage $t$, bidders receive rebates of their initial cost shares determined by the function

$$
R_{i}^{t}\left(s_{i}, b^{t}\right)= \begin{cases}s_{i} c & \text { if } \sum b_{j}^{t}<c \\ 0 & \text { otherwise }\end{cases}
$$

and a pay a incentive tax determined by the function

$$
\tau_{i}^{t}\left(s_{i}, b_{-i}^{t}\right)=\left\{\begin{array}{ll}
\max \left\{0,\left(s_{i}-1\right) c+\sum_{j \neq i} b_{j}^{t}\right\} & \text { if } \sum b_{j}^{t}<c \\
\max \left\{0,\left(1-s_{i}\right) c-\sum_{j \neq i} b_{j}^{t}\right\} & \text { otherwise }
\end{array} .\right.
$$


Our next theorem shows truth telling in the DQ-CAA is an ex-post Nash equilibrium.

Theorem 4 In the full bid information DQ-CAA, truth telling by each bidder is an ex-post Nash equilibrium. Moreover, the equilibrium production level is efficient and the tax revenue generated by the auction covers the cost of production.

Since the auction is descending, the highest valuations are preserved. Truth telling is an equilibrium so the equilibrium production level of $x$ is efficient by design. Finally, we are assured costs are covered when the auction ends. This follows from the fact that costs are covered when the auction begins, incentive taxes accrued during the auction are positive, and rebates are only given for units that are not produced. It is straight forward to verify that the outcome of this auction is equivalent to Clarke (1971). ${ }^{11}$ Other results such as the analogs to Corollary 1 and Theorem 3 can also be shown to hold for the DQ-CAA as well.

\section{Conclusion}

This paper has shown that the relationship between the dynamic private Ausubel auction and the static Vickrey auction can be extended to public good environments in a natural way by exploiting the dual nature of the private good/ public good problem. Once the description of the Ausubel auction has been augmented to fit the new public good environment and the appropriate assumptions are introduced many of Ausubel's same results apply. The auction seems simple, transparent, scales well to increases in the number of bidders. Moreover, strategic incentives can be eliminated by restricting information during the auction. However, the AQ-AA does not protect the highest valuations of bidders or raise enough tax revenue to finance production. The DQCAA remedies both of these issues and has strategic properties that are similar to the AQ-AA. Whether one of these mechanisms is more desirable in practice is an empirical question and one that is well posed for future experimental research.

\section{Appendix}

Proof of Theorem 2 Suppose the profile of truthful bidding strategies $\beta$ is not an expost perfect Nash equilibrium. Then there exists $\bar{\theta} \in \Theta$ such that the projection of strategy profile $\beta$ to $\Gamma^{e}(\bar{\theta})$, hereafter $\delta$, is not subgame perfect. Since $\bar{x}<\infty$ and there is "full bid information," $\Gamma^{e}(\bar{\theta})$ is a finite horizon game with observed action. Since $\delta$ is not subgame perfect it does not satisfy the "one-stage-deviation principle" for finite horizon games. ${ }^{12}$ Thus, there is some bidder $i$ and strategy $\tilde{\delta}_{i}$ that agrees with $\delta_{i}$ except at a single $t$ and $h^{t}$, where $\tilde{\delta}_{i}$ is a better response to $\delta_{-i}$ than $\delta_{i}$ conditional on history $h^{t}$ being reached. Suppose conditional on stage $t$ being reached the auction ends at stage $L \geq t$ (i.e., $x^{*}=L-1$ ) if all bidders report truthfully. Given the truthful reports of the other bidders, a 1 stage deviation in the AQ-AA at stage $t$ can only result in three outcomes: Case 1, the deviation doesn't change the outcome (i.e., the auction

\footnotetext{
11 See also, Tideman and Tullock (1976) for a discussion about the Clarke mechanism and its properties.

12 See, for instance, Fudenberg and Tirole (1991) p. 109.
} 
ends at $L$ ); Case 2, the auction ends earlier at $t<L$; Case 3, the auction ends in round $L+1$ (i.e., $t=L$ ). Case 1 is obviously not a profitable deviation since the auction ends at the same round and bidder $i$ 's payment is independent of his own action. Suppose Case 2 is true, then the one stage deviation causes the auction to end earlier than $L$ say round $t=E\left(x^{*}=E-1\right)$. Bidder $i$ 's payoff is $v_{i}\left(E-1, \theta_{i}\right)-\tau_{i}\left(E, b_{-i}\right)$. The payoff from truth telling is $v_{i}\left(E-1, \theta_{i}\right)-\tau_{i}\left(E, b_{-i}\right)+\sum_{k=E}^{L-1}\left[\theta_{i}^{k}-\tilde{s}_{i}\left(k, b_{-i}^{k}\right)\right]$. For each $k \in\{E, \ldots, L-1\}$ it must be true that $\sum_{j=1}^{N} \theta_{j}^{k} \geq c$, since otherwise the auction would have ended earlier when everyone was truthfully reporting. However, this implies that $\theta_{i}^{k}>c-\sum_{j \neq i} \theta_{j}^{k}$. Since $\theta_{i}^{k} \geq 0$, by assumption, we also have $\theta_{i}^{k} \geq \max \left\{0, c-\sum_{j=1}^{N} \theta_{j}^{k}\right\}=\tilde{s}_{i}\left(k, b_{-i}^{k}\right)$. Thus, for each $k$, we have $\theta_{i}^{k} \geq \tilde{s}_{i}\left(k, b_{-i}^{k}\right)$ which in turn implies $\sum_{k=E}^{L-1}\left[\theta_{i}^{k}-\tilde{s}_{i}\left(k, b_{-i}^{k}\right)\right] \geq 0$. Therefore ending the auction before $L$ cannot lead to a profitable deviation. Suppose Case 3 is true, then the one stage deviation causes the auction to end at round $L+1$ (i.e., $x^{*}=L$ being produced). ${ }^{13}$ Bidder $i$ 's payoff from this deviation is $v_{i}\left(L-1, \theta_{i}\right)+\theta_{i}^{L}-\tau^{i}\left(L, b_{-i}\right)-\tilde{s}_{i}\left(L, b_{-i}^{L}\right)$. Bidder $i$ 's payoff from truthful reporting is $v_{i}\left(L-1, \theta_{i}\right)-\tau_{i}\left(L, b_{-i}\right)$. The single stage deviation is profitable only if and only if $\theta_{i}^{L}-\tilde{s}_{i}\left(L, b_{-i}^{L}\right)>0$. However, when bidders were bidding truthfully the auction stopped at $L$. From the continuation rule of the AQ-AA, it must be that $\sum_{i} \theta_{i}^{L}<c$ or $0 \leq \theta_{i}^{L}<c-\sum_{j \neq i} \theta_{j}^{L}=s_{i}\left(L, b_{-i}^{L}\right)$, which contradicts claim truthful revelation was not subgame perfect in the realized game. Thus, $\beta$ is ex-post perfect. It follows that equilibrium outcomes of the AQ-AA and Vickrey auctions under truth telling are the same.

Proof of Corollary 1 First, we look at the aggregate bid information AQ-AA. For each $i$, consider the 2 bidder game between $i$ and a representative agent for the other $N-1$ bidders whose value at each stage is the sum of the $N-1$ bidders' values whom he represents. This is a 2 bidder AQ-AA with full bid information. From Theorem 2, we know that truth telling is an ex-post perfect Nash equilibrium. Therefore, for every realization of types, given aggregate bid information at each stage of the auction and truth telling of the $N-1$ other agents, truth telling is a best response. Since this is true for all players at each stage of the auction, it is true at each subgame. Thus, truth telling is an ex-post perfect Nash equilibrium. The no bid information case follows similarly.

Proof of Theorem 3 Let $\hat{\beta}_{i}$ be $i$ 's truth telling strategy. Since no bid information is being given, no bidder can distinguish between 'auction continuing' strategies of their rivals. So at any information set where the auction is continued, no bidder knows whether or not they were pivotal at any specific rounds. Suppose that given $\left(\hat{\beta}_{i}, \beta_{-i}\right)$ the auction ends at round $L$ yielding $i$ a payoff of $u_{i}\left(\hat{\beta}_{i}, \beta_{-i}\right)$ and consider a deviation to $\beta_{i} \neq \hat{\beta}_{i}$. If $\beta_{i}$ ends the auction in round $L$, then it gives $i$ the same payoff since $i$ 's tax at any given round is independent of $i$ 's action. Since the other bidders can't distinguish between $\beta_{i}$ and $\hat{\beta}_{i}$ for rounds 1 to $L$, they cannot respond to the change. If $\beta_{i}$ ends the auction in round $E<L$. Bidder $i$ 's surplus for the first $E-1$ rounds is exactly the same as when he was bidding truthfully so there are no gains for those

\footnotetext{
13 That the deviation has to come at this stage follows from the assumption of weakly decreasing marginal valuations and the fact that $\tilde{b}_{i}$ deviates from $b_{i}$ in only one stage.
} 
units. Furthermore, since truth telling guarantees non-negative surplus at each round, bidder $i$ is potentially foregoing positive payoffs from round $E$ to $L-1$. Last, if $\beta_{i}$ ends the auction in round $M>L$, then $i$ 's surplus for the first $L-1$ rounds is exactly the same as under $\hat{\beta}_{i}$. This is since, by the "No Bid Information" constraint, bidders other than $i$ cannot respond to the change and the fact that $i$ 's tax is independent of own action. Since the auction ended in round $L$ when $i$ was bidding truthfully $\theta_{i}^{L}<c-\sum_{j \neq i} b_{j}^{L}$. Therefore the tax for changing $i$ 's bid in round $L$ is bigger than the gains from having the auction continuing for that round. Gains in later rounds may offset the losses from this round. However, this cannot happen. Bids are decreasing so $c-\sum_{j \neq i} b_{j}^{t}$ is a weakly increasing function in $t$. Marginal valuations are weakly decreasing. Thus, $i$ 's payoff from continuing past round $L$ is strictly decreasing-i.e., $u_{i}\left(\beta_{i}, \beta_{-i}\right)<u_{i}\left(\hat{\beta}_{i}, \beta_{-i}\right)$. Thus, for all $\beta_{-i}, u_{i}\left(\hat{\beta}_{i}, \beta_{-i}\right) \geq u_{i}\left(\beta_{i}, \beta_{-i}\right)$. The second part of the proof follows Claim 2 in Van Essen (2010).

Proof of Theorem 4 Suppose $x^{*}$ is the level of production under truth telling and consider a deviation by bidder $i$. Because the other bidders are truth telling and since $i$ 's bid must be increasing, the only changes in $i$ 's payoff with a deviation occur at the changed units of $x$-i.e., if the deviation doesn't change $x^{*}$, there is no difference in payoff and deviation is not profitable. Suppose $x^{*}$ is increased to $\hat{x}$ from $i$ 's deviation. Then $i$ is not refunded for the $\hat{x}-x^{*}$ units and the tax for the first $x^{*}$ units is the same. Two cases need to be considered. First, if $s_{i} c \geq \theta_{i}^{k}$, the added benefit $\sum_{i} \theta_{i}^{k}+\left(s_{i}-1\right) c$ is smaller than the refund $c+\left(s_{i}-1\right) c=s_{i} c$ since $\sum_{i} \theta_{i}^{k}<c$. Second, if $s_{i} c \leq$ $\theta_{i}^{k}$, for each additional unit $k$, then since, $x^{*}<\hat{x}$, we have $\theta_{i}^{k} \leq c-\sum_{j \neq i} \theta_{j}^{k}$. Thus, for each unit $k, \theta_{i}^{k}-s_{i} c \leq\left(1-s_{i}\right) c-\sum_{j \neq i} \theta_{j}^{k}-$ i.e., any extra value the bidder gets from unit $k$ is smaller than additional tax. So, neither of these cases results in a profitable deviation. Last, suppose the deviation causes $x$ to be decreased to $\check{x}$. Now the tax is the same for the first $\check{x}$ units and $i$ is refunded for the $x^{*}-\check{x}$ units. Again, two cases need to be considered. First, suppose $s_{i} c \leq \theta_{i}^{k}$ for each removed unit $k$. Since, $x^{*}>\check{x}$, we have $\theta_{i}^{k} \geq c-\sum_{j \neq i} \theta_{j}^{k}$ which implies the value lost $\theta_{i}^{k}$ is larger than $s_{i} c+c\left(1-s_{i}\right)-\sum_{j \neq i} \theta_{j}^{k}=c-\sum_{j \neq i} \theta_{j}^{k}$, or the fiscal savings for the removed units. Second, suppose $s_{i} c \geq \theta_{i}^{k}$, for each removed unit $k$. Since, $x^{*}>\check{x}$, all the removed units $k$ are such that $\theta_{i}^{k} \geq c-\sum_{j \neq i} \theta_{j}^{k}$. Thus, we have $\left(s_{i}-1\right) c+\sum_{j \neq i} \theta_{j}^{k} \geq \theta_{i}^{k}$ $-\left(c-\sum_{j \neq i} \theta_{j}^{k}\right) \geq \theta_{i}^{k}-s_{i} c \geq 0-$ i.e., the new loss for unit $k$ is larger than the original loss for unit $k$. Therefore, there are no profitable deviations for $i$. Since this argument hold for any $\theta_{-i}$, truth telling is an ex-post Nash equilibrium.

\section{References}

Ausubel, L.: An efficient ascending-bid auction for multiple objects. Am. Econ. Rev. 94(5), 1452-1475 (2004)

Bergemann, D., Välimäki, J.: Dynamic Pivot mechanism. Econometrica 78, 771-789 (2010)

Clarke, E.: Multipart pricing of public goods. Publ. Choice 11(1), 17-33 (1971)

Cremer, J., McLean, R.: Optimal selling strategies under uncertainty for a discriminating monopolist when demands are interdependent. Econometrica 53(2), 345-361 (1985)

Dreze, J., Pousin, D.: A tâ tonnement process for public goods. Rev. Econ. Stud. 38, 133-150 (1971)

Fudenberg, D., Tirole, J.: Game Theory. The MIT Press, Cambridge (1991) 
Groves, T.: Incentives in teams. Econometrica 41(4), 617-631 (1973)

Holmstrom, B., Myerson, R.: Efficient and durable decision rules with incomplete information. Econometrica 51, 1799-1820 (1983)

Kagel, J., Levin, D.: Behavior in multi-unit demand auctions: experiments with uniform price and dynamic Vickrey auctions. Econometrica 69(2), 413-454 (2001)

Kagel, J., Pevnitskaya, S.: Survival auctions. Econ. Theory 33(1), 103-119 (2007)

Loeb, M.: Alternative versions of the demand-revealing process. Publ. Choice 29, 15-26 (1977)

Malinvaud, E.: A planning approach to the public good problem. Swed. J. Econ. 73, 96-112 (1971)

Milgrom, P.: Putting Auction Theory to Work, Cambridge (2004)

Rothkopf, M.: Thirteen reasons why the Vickrey-Clarke-Groves process is not practical. Oper. Res. 55(2), 191-197 (2007)

Tideman, N., Tullock, G.: A new and superior process for making social choices. J. Polit. Econ. 84(6), $1145-1159$ (1976)

Van Essen, M. (2010) Making efficient public good decisions using an augmented Ausubel auction. University of Alabama Working Paper WP10-06-03

Vickrey, W.: Counterspeculation, auctions, and competitive sealed-tenders. J. Finance 16, 8-37 (1961) 